\title{
EXPONENTS OF CARTESIAN PRODUCTS OF TWO DIGRAPHS OF SPECIAL ORDERS
}

\author{
Byeong Moon Kim And Yoomi Rho
}

\begin{abstract}
In this paper, we find the maximum exponent of $D \times E$, the cartesian product of two digraphs $D$ and $E$ on $n, n+2$ vertices, respectively for an even integer $n \geq 4$. We also characterize the extremal cases.
\end{abstract}

\section{Introduction}

Let $D=(V, A)$ be a digraph on $n$ vertices and $u, v \in V . \mathrm{A} u \rightarrow v$ walk is a walk from $u$ to $v$. We use the notation $u \stackrel{k}{\longrightarrow} v$ when there is a $u \rightarrow v$ walk of length $k$. A digraph $D=(V, A)$ is said primitive if for some $k, u \stackrel{k}{\longrightarrow} v$ for all pair of vertices $u, v$ of $D$. In this case, the smallest such $k$ is called the exponent of $D$ and denoted by $\exp (D)$. For a matrix $A$, the minimal $k$ such that all the entries of $A^{k}$ are positive is called the exponent of $A$. The exponent of a primitive digraph $D$ is equal to the exponent of its adjacency matrix. Wielandt [7] found that the maximum exponent of primitive digraphs on $n$ vertices is $W_{n}=n^{2}-2 n+2$ and characterized all the digraphs attaining this bound, which are called Wielandt graphs. Shao [6] improved this bound to $2 n-2$ and Liu, McKay, Wormald and Zhang [4] characterized all the digraphs attaining this improved bound.

Let $D=\left(V_{D}, A_{D}\right)$ and $E=\left(V_{E}, A_{E}\right)$ be digraphs such that $\left|V_{D}\right|=n$, $\left|V_{E}\right|=m$. The cartesian product of $D$ and $E$ is defined as $D \times E=(V, A)$ where $V=V_{D} \times V_{E}$ and

$$
\begin{aligned}
A=\left\{\left(\left(u_{1}, v_{1}\right),\left(u_{2}, v_{2}\right)\right) \mid\right. & \left(\left(u_{1}, u_{2}\right) \in A_{D} \quad \text { and } v_{1}=v_{2}\right) \quad \text { or } \\
& \left.\left(u_{1}=u_{2} \text { and }\left(v_{1}, v_{2}\right) \in A_{E}\right)\right\} .
\end{aligned}
$$

R. Lamprey and B. Barnes [3] showed that if $D \times E$ is primitive, then

$$
\exp (D \times E) \leq(n+m)^{2}-4(n+m)+5 .
$$

Received January 13, 2009

2000 Mathematics Subject Classification. 05C20, 05C38, 05C50, 15A48.

Key words and phrases. exponents, digraphs, Cartesian products, Wielandt graphs, Frobenius numbers.

This work is supported by the University of Incheon research grant in 2006-2007. 
The first author, Song and Hwang [2] improved this upper bound as

$$
\exp (D \times E) \leq n m-1 \text {. }
$$

They also showed that the upper bound in (1) is attained when $(m, n)=1$ and characterized all the digraphs $D$ and $E$ which attain it. Moreover, when $m=n$, they proved that

$$
\exp (D \times E) \leq n^{2}-n+1
$$

where the equality holds if and only if $D$ and $E$ are isomorphic to a directed cycle and a Wielandt graph. In this paper, we prove that if $D$ and $E$ are digraphs on $n$ and $n+2$ vertices respectively for an even integer $n \geq 4$, and $D \times E$ is primitive, then

$$
\exp (D \times E) \leq n^{2}+n
$$

and we characterize all the extremal digraphs.

\section{Main result}

From now on we assume that $D=\left(V_{D}, A_{D}\right)$ and $E=\left(V_{E}, A_{E}\right)$ are digraphs on $n$ and $m$ vertices, respectively and $D \times E$ is primitive. Let $l_{1}$ be the smallest length of a directed cycle of $D$ and $l_{i}$ be the smallest length of a directed cycle of $D$ which is not a multiple of $\left(l_{1}, \ldots, l_{i-1}\right)$ for $i \geq 2$. Let $h$ be the last index of such $i$. Now let $l_{j}$ be the smallest length of a directed cycle of $E$ which is not a multiple of $\left(l_{1}, \ldots, l_{j-1}\right)$ for $j \geq h+1$. Let $k$ be the last index of such $j$. For $1 \leq i \leq h$, let $d_{i}=\left(l_{1}, \ldots, l_{i}\right)$ and $C_{i}$ be a directed cycle of $D$ whose length is $l_{i}$. For $1 \leq j \leq k$, let $d_{j}=\left(l_{1}, \ldots, l_{j}\right)$ and $C_{j}$ be a directed cycle of $E$ whose length is $l_{j}$. Since $D \times E$ is primitive, $k \geq 2, d_{k}=1$ and $\frac{d_{1}}{d_{2}}, \frac{d_{2}}{d_{3}}, \ldots, \frac{d_{k-1}}{d_{k}} \geq 2$.

For relatively prime positive numbers $l_{1}, \ldots, l_{k}$, the Frobenius number $g\left(l_{1}\right.$, $\left.l_{2}, \ldots, l_{k}\right)$ is the largest number $G$ such that the equation $l_{1} x_{1}+\cdots+l_{k} x_{k}=G$ is not solvable for non-negative integers $x_{1}, \ldots, x_{k}$. Classical results on Frobenius numbers are as follows.

Lemma 1 ([1]). For relatively prime positive numbers $l_{1}, \ldots, l_{k}$,

$$
g\left(l_{1}, l_{2}, \ldots, l_{k}\right) \leq l_{2} \frac{d_{1}}{d_{2}}+l_{3} \frac{d_{2}}{d_{3}}+\cdots+l_{k} \frac{d_{k-1}}{d_{k}}-l_{1}-l_{2}-\cdots-l_{k},
$$

where $d_{i}=\left(l_{1}, \ldots, l_{i}\right)$ for $1 \leq i \leq k$.

Lemma 2 ([1, 5$])$. If $(a, d)=1$,

$$
g(a, a+d, a+2 d, \ldots, a+k d)=\left(\left\lfloor\frac{a-2}{k}\right\rfloor+d\right) a-d .
$$

The followings are from the first author, Song and Hwang [2].

Lemma $3([2])$. Let $D$ and $E$ be digraphs on $n$ and $m$ vertices, respectively with $h, k, l_{1}, \ldots, l_{k}$ as above. Then

$$
\exp (D \times E) \leq g\left(l_{1}, l_{2}, \ldots, l_{k}\right)-l_{1}-\cdots-l_{k}+(h+1) m+(k-h+1) n-1 .
$$


Lemma 4 ([2]). Let $D$ and $E$ be digraphs on $n$ and $m$ vertices, respectively with $k$ as above. If $k \geq 3$, then

$$
\exp (D \times E) \leq \frac{n m}{2}+m-1 .
$$

Assume $k \geq 4$. Let $V_{k}=\{0,1, \ldots, k-1\},\left(A_{0}\right)_{k}=\{(i, i+1) \mid 0 \leq i \leq$ $k-2\} \cup\{(k-2,0)\}$ and $\tilde{B}_{k}=\{(k-1,1),(k-1,2),(k-2,1),(k-3,0)\}$ and $\tilde{E}_{k}=\left(V_{k}, \tilde{A_{k}}\right)$ be a digraph where $\tilde{A}_{k}=\left(A_{0}\right)_{k} \cup \tilde{B_{k}}$ as shown in Figure 1. Also let $\mathcal{E}_{k}$ be the set of digraphs $E_{k}=\left(V_{k}, A_{k}\right)$ such that $A_{k}=\left(A_{0}\right)_{k} \cup B_{k}$ where $B_{k}$ is a subset of $\tilde{B}_{k}$ which contains at least one of $(k-1,1)$ or $(k-1,2)$. Then $\tilde{E}_{k} \in \mathcal{E}_{k}$ and every element of $\mathcal{E}_{k}$ is a subgraph of $\tilde{E}_{k}$. Note that $Z_{n}=$ $\left(V_{n},\{(i, i+1) \mid 0 \leq i \leq n-2\} \cup\{(n-1,0)\}\right)$.

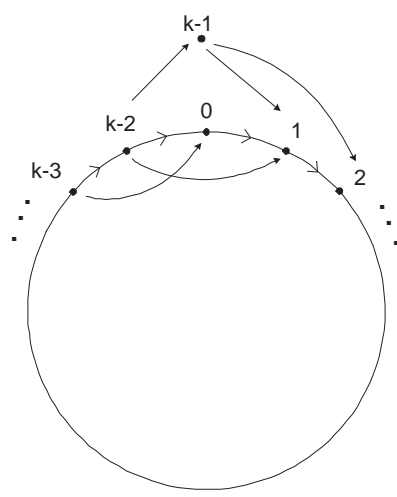

Figure 1

Lemma 5. Let $E=(V, A)$ be a digraph with $m$ vertices and $v, w \in V$. If the distance from $v$ to $w$ is $m-1$ and $l_{1}, l_{2}, \ldots, l_{k}$ are all the lengths of directed cycles in $E$, then each length of a path from $v$ to $w$ is represented by $m-1+$ $l_{1} x_{1}+l_{2} x_{2}+\cdots+l_{k} x_{k}$ where $x_{1}, x_{2}, \ldots, x_{k}$ are nonnegative integers.

Proof. If there is a path from $v$ to $w$ whose length is not of the form $m-1+$ $l_{1} x_{1}+l_{2} x_{2}+\cdots+l_{k} x_{k}$, then we can choose a path whose length is minimal among the paths with this property. Let $v=v_{0} \rightarrow v_{1} \rightarrow \cdots \rightarrow v_{t}=w$ be such a path. If there are no repeated vertices among $v_{0}, v_{1}, \ldots, v_{t}$, then $t+1 \leq m$. While considering that the distance from $v$ to $w$ is $m-1, t \geq m-1$ and hence $t=m-1$. This is a contradiction. If there are repeated vertices among $v_{0}, v_{1}, \ldots, v_{t}$, then we can take a pair $i, j$ such that $0 \leq i<j \leq t, v_{i}=v_{j}$ and $j-i$ is minimal among the pairs with this property. Then $v_{i} \rightarrow v_{i+1} \rightarrow \cdots \rightarrow v_{j}=v_{i}$ is a directed cycle, $j-i=l_{h}$ for some $1 \leq h \leq k$. Therefore

$$
v=v_{0} \rightarrow v_{1} \rightarrow \cdots \rightarrow v_{i}=v_{j} \rightarrow v_{j+1} \rightarrow \cdots \rightarrow v_{t}=w
$$

is a path from $v$ to $w$ with length $t-j+i<t$. By the minimality of $t$, $t-j+i=t-l_{h}=m-1+l_{1} x_{1}+l_{2} x_{2}+\cdots+l_{k} x_{k}$ for some nonnegative integers 
$x_{1}, x_{2}, \ldots, x_{k}$ and hence $t=m-1+l_{1} x_{1}+l_{2} x_{2}+\cdots+l_{h-1} x_{h-1}+l_{h}\left(x_{h}+1\right)+$ $l_{h+1} x_{h+1}+\cdots+l_{k} x_{k}$. This is a contradiction. Thus the lemma is proved.

Lemma 6. Let $E=(V, A)$ be a digraph on $n+2$ vertices. If $Z_{n} \times E$ is primitive, $\operatorname{diam}(E)=n+1$ and all the lengths of directed cycles in $E$ is $n$ and $n+1$, then

$$
\exp \left(Z_{n} \times E\right) \geq n^{2}+n
$$

Proof. Since $\operatorname{diam}(E)=n+1$, there are $v, w \in V$ such that the distance from $v$ to $w$ is $n+1$. It is enough to show that $(0, v) \stackrel{n^{2}+n-1}{\rightarrow}(n-1, w)$. Suppose $(0, v) \stackrel{n^{2}+n-1}{\longrightarrow}(n-1, w)$. Then $(0, v)=\left(u_{0}, v_{0}\right) \longrightarrow\left(u_{1}, v_{1}\right) \longrightarrow$ $\cdots \longrightarrow\left(u_{n^{2}+n-1}, v_{n^{2}+n-1}\right)=(n-1, w)$ for some vertices $\left(u_{i}, v_{i}\right)$ of $Z_{n} \times E$. Let

$S=\left\{i \mid 1 \leq i \leq n^{2}+n-1, u_{i-1} \neq u_{i}\right\}$ and $T=\left\{i \mid 1 \leq i \leq n^{2}+n-1, v_{i-1} \neq v_{i}\right\}$ with $|S|=s$ and $|T|=t$. Then $S \cup T=\left\{i \mid 1 \leq i \leq n^{2}+n-1\right\} \quad$ and $\quad S \cap T=\phi$. Therefore $s+t=n^{2}+n-1$. By Lemma $5, s=n-1+n x$ and $t=n+1+$ $n y+(n+1) z$ for some nonnegative integers $x, y, z$. Thus $n^{2}+n-1=s+t=$ $n-1+n x+n+1+n y+(n+1) z$ and hence $n^{2}-n-1=n(x+y)+(n+1) z$. Considering $g(n, n+1)=n^{2}-n-1$, this is impossible.

Theorem 1. If $E=(V, A) \in \mathcal{E}_{n+2}$, then

$$
\exp \left(Z_{n} \times E\right) \geq n^{2}+n \text {. }
$$

Proof. Considering that $0 \stackrel{n}{\longrightarrow} n \rightarrow n+1, \operatorname{diam}(E)=n+1 . Z_{n} \times E_{n+2}$ is primitive as $Z_{n}$ and $E_{n+2}$ contain cycle of length $n$ and $n+1$, respectively. Also $E_{n+2}$ contains directed cycles of length $n$ and $n+1$ only. Thus by Lemma 6 , the theorem is proved.

Definition 1. Let $D=(V, A)$ be a digraph. Denote $A^{T}=\{(v, w) \mid(w, v) \in A\}$. we call a digraph $\left(V, A^{T}\right)$ the transpose of $D$ and denote it by $D^{T}$.

Remark 1. $\exp (D)=\exp \left(D^{T}\right)$.

Theorem 2. Let $n \geq 4$ be even. Assume $D$ and $E$ are digraphs on $n$ and $n+2$ vertices respectively and $D \times E$ is primitive. Then

$$
\exp (D \times E) \leq n^{2}+n
$$

and

(ii) the equality holds if and only if $D$ is isomorphic to $Z_{n}$, and $E$ or $E^{T}$ belongs to $\mathcal{E}_{n+2}$.

Proof. (i) Take $h, k, l_{1}, \ldots, l_{k}$ as above. If $k \geq 3$, then from Lemma 4 ,

$$
\exp (D \times E) \leq \frac{n(n+2)}{2}+n+2-1<n^{2}+n .
$$


Assume that $k=2$. From Lemma 1 and Lemma 3,

$$
\begin{aligned}
\exp (D \times E) & \leq g\left(l_{1}, l_{2}\right)-l_{1}-l_{2}+2 n+2(n+2)-1 \\
& \leq l_{1} l_{2}-2 l_{1}-2 l_{2}+4 n+3 \\
& =\left(l_{1}-2\right)\left(l_{2}-2\right)+4 n-1 .
\end{aligned}
$$

If $l_{1} \leq n-1$, then $\exp (D \times E) \leq n^{2}+n-1$ as $l_{2} \leq n+2$. Assume that $l_{1}=n$. Then $D$ is isomorphic to the directed cycle of length $n$, which is $Z_{n}$. Since $\left(l_{1}, l_{2}\right)=1, l_{2}=n+1$ and hence

$$
\exp (D \times E) \leq(n-2)(n-1)+4 n-1=n^{2}+n+1 .
$$

Assume that $C_{1}$ is a directed cycle $u_{0} \rightarrow u_{1} \rightarrow \cdots \rightarrow u_{n-1} \rightarrow u_{0}$ in $D$ and $C_{2}$ is a directed cycle $v_{0} \rightarrow v_{1} \rightarrow \cdots \rightarrow v_{n} \rightarrow v_{0}$ in $E$. Let $\left(x_{1}, y_{1}\right)$ and $\left(x_{2}, y_{2}\right)$ be vertices on $D \times E$ such that $\left(x_{1}, y_{1}\right) \stackrel{\alpha-1}{\nrightarrow}\left(x_{2}, y_{2}\right)$ where $\alpha=\exp (D \times E)$. Then there are integers $s$ and $t$ such that $x_{1} \stackrel{s}{\longrightarrow} x_{2}, y_{1} \stackrel{t}{\longrightarrow} y_{2}, 0 \leq s \leq n-1$ and $0 \leq t \leq n+1$. We consider the following two cases.

Case (i)a: There is a directed cycle of length $n+2$ in $E$.

Let $\beta \geq n^{2}+n-1$. If $y_{1} \neq y_{2}$, then at least one of $y_{1}, y_{2}$ belongs to the directed cycle of length $n+1$. We may assume that $y_{1}$ does and hence $y_{1} \stackrel{n+1}{\longrightarrow} y_{1}$. By Lemma 2,

$$
\begin{aligned}
\beta-s-t & \geq n^{2}+n-1-(n-1)-(n+1)=n^{2}-n-1 \\
& >\frac{n^{2}}{2}-1=g(n, n+1, n+2)
\end{aligned}
$$

and hence there are $p, q, r \geq 0$ such that $n p+(n+1) q+(n+2) r=\beta-s-t$. Then, since

$$
\left(x_{1}, y_{1}\right) \stackrel{n p}{\longrightarrow}\left(x_{1}, y_{1}\right) \stackrel{s}{\longrightarrow}\left(x_{2}, y_{1}\right) \stackrel{(n+1) q}{\longrightarrow}\left(x_{2}, y_{1}\right) \stackrel{t}{\longrightarrow}\left(x_{2}, y_{2}\right) \stackrel{(n+2) r}{\longrightarrow}\left(x_{2}, y_{2}\right)
$$

and $n p+s+(n+1) q+t+(n+2) r=(\beta-s-t)+s+t=\beta,\left(x_{1}, y_{1}\right) \stackrel{\beta}{\longrightarrow}\left(x_{2}, y_{2}\right)$. So $\exp (D \times E) \leq n^{2}+n-1$. If $y_{1}=y_{2}$, then $y_{1} \stackrel{t_{1}}{\longrightarrow} v_{0} \stackrel{t_{2}}{\longrightarrow} y_{1}$ for some $0 \leq t_{1}, t_{2} \leq n+1$ such that $t_{1}+t_{2}=n+2$ as $E$ is strongly connected.

$$
\begin{aligned}
\beta-s-(n+2) & \geq n^{2}+n-1-(n-1)-(n+2)=n^{2}-n-2 \\
& >\frac{n^{2}}{2}-1=g(n, n+1, n+2)
\end{aligned}
$$

by Lemma 2 . So there are $p, q, r \geq 0$ such that $n p+(n+1) q+(n+2) r=$ $\beta-s-(n+2)$. Then, since

$$
\begin{aligned}
& \left(x_{1}, y_{1}\right) \stackrel{n p}{\longrightarrow}\left(x_{1}, y_{1}\right) \stackrel{s}{\longrightarrow}\left(x_{2}, y_{1}\right) \stackrel{t_{1}}{\longrightarrow}\left(x_{2}, v_{0}\right) \stackrel{(n+1) q}{\longrightarrow}\left(x_{2}, v_{0}\right) \\
& \stackrel{t_{2}}{\longrightarrow}\left(x_{2}, y_{1}\right) \stackrel{(n+2) r}{\longrightarrow}\left(x_{2}, y_{1}\right)
\end{aligned}
$$

and $n p+s+t_{1}+(n+1) q+t_{2}+(n+2) r=(\beta-s-(n+2))+s+n+2=\beta$, $\left(x_{1}, y_{1}\right) \stackrel{\beta}{\longrightarrow}\left(x_{2}, y_{1}\right)$. So $\exp (D \times E) \leq n^{2}+n-1$. 
Case (i)b: There is no directed cycle of length $n+2$ in $E$.

If $t \geq 1$, then since there is only one vertex $x \in V_{E}$ such that $x \neq v_{i}$ for $i=0,1 \ldots, n$, there is an intermediate vertex $v_{j}$ such that $y_{1} \stackrel{t_{1}}{\longrightarrow} v_{j} \stackrel{t-t_{1}}{\longrightarrow} y_{2}$. Since

$n^{2}+n-s-t \geq n^{2}+n-(n-1)-(n+1)=n^{2}-n>(n-1) n-1=g(n, n+1)$, there are $p, q \geq 0$ such that $n p+(n+1) q=n^{2}+n-s-t$. Then, since

$$
\left(x_{1}, y_{1}\right) \stackrel{n p}{\longrightarrow}\left(x_{1}, y_{1}\right) \stackrel{s}{\longrightarrow}\left(x_{2}, y_{1}\right) \stackrel{t_{1}}{\longrightarrow}\left(x_{2}, v_{j}\right) \stackrel{(n+1) q}{\longrightarrow}\left(x_{2}, v_{j}\right) \stackrel{t-t_{1}}{\longrightarrow}\left(x_{2}, y_{2}\right)
$$

and $n p+s+t_{1}+(n+1) q+t-t_{1}=\left(n^{2}+n-s-t\right)+s+t=n^{2}+n$, $\left(x_{1}, y_{1}\right) \stackrel{n^{2}+n}{\longrightarrow}\left(x_{2}, y_{2}\right)$. If $t=0$, then $y_{1}=y_{2}$. As $E$ is strongly connected, $y_{1} \stackrel{t_{1}}{\longrightarrow} v_{0} \stackrel{t_{2}}{\longrightarrow} y_{1}$ for some $0 \leq t_{1}, t_{2} \leq n+1$ such that $t_{1}+t_{2} \leq n+1$. Since $n^{2}+n-s-\left(t_{1}+t_{2}\right) \geq n^{2}+n-(n-1)-(n+1)=n^{2}-n>n^{2}-n-1=g(n, n+1)$, there are $p, q \geq 0$ such that $n p+(n+1) q=n^{2}+n-s-\left(t_{1}+t_{2}\right)$. Then, since

$$
\left(x_{1}, y_{1}\right) \stackrel{n p}{\longrightarrow}\left(x_{1}, y_{1}\right) \stackrel{s}{\longrightarrow}\left(x_{2}, y_{1}\right) \stackrel{t_{1}}{\longrightarrow}\left(x_{2}, v_{0}\right) \stackrel{(n+1) q}{\longrightarrow}\left(x_{2}, v_{0}\right) \stackrel{t_{2}}{\longrightarrow}\left(x_{2}, y_{1}\right)
$$

and $n p+s+t_{1}+(n+1) q+t_{2}=\left(n^{2}+n-s-\left(t_{1}+t_{2}\right)\right)+s+t_{1}+t_{2}=n^{2}+n$, $\left(x_{1}, y_{1}\right) \stackrel{n^{2}+n}{\longrightarrow}\left(x_{2}, y_{2}\right)$. So $\exp (D \times E) \leq n^{2}+n$.

(ii) Now we characterize all the extremal graphs. Let $\exp (D \times E)=n^{2}+n$. It is possible only in Case (i)b where $E$ has no directed cycle of length $n+2$. If $s+t \leq 2 n-1$, then similarly as above, $\exp (D \times E) \leq n^{2}+n-1$. So $s=n-1$ and $t=n+1$. Let $\left(x_{1}, y_{1}\right)$ and $\left(x_{2}, y_{2}\right)$ be vertices of $D \times E$ such that $x_{1} \stackrel{s}{\longrightarrow} x_{2}, y_{1} \stackrel{t}{\longrightarrow} y_{2}$. Also let $w_{0}$ be the only vertex of $E$ such that $w_{0} \neq v_{i}$ for all $i=0,1, \ldots, n$. Since $E$ is strongly connected, there are $i, j$ such that $\left(v_{i}, w_{0}\right),\left(w_{0}, v_{j}\right) \in A_{E}$. So we may also assume $\left(v_{n}, w_{0}\right) \in A_{E}$. As $w_{0} \rightarrow v_{j} \rightarrow$ $v_{j+1} \rightarrow \cdots \rightarrow v_{n} \rightarrow w_{0}$ is a directed cycle of length $n \leq n+2-j<n+2, j$ is 1 or 2 .

Case (ii)a: $j=1$.

As there can be only directed cycles of order $n$ or $n+1$ in $E$, among the arcs which are adjacent to $w_{0}$, at most one of $\left(w_{0}, v_{2}\right),\left(v_{n-1}, w_{0}\right)$ may exist. Since $y_{1} \stackrel{n+1}{\longrightarrow} y_{2},\left(y_{1}, y_{2}\right)$ is $\left(v_{0}, w_{0}\right)$ in the first case, and $\left(w_{0}, v_{0}\right)$ in the second case. Let $0 \leq k<l \leq n$. If $\left(v_{l}, v_{k}\right) \in A_{E}$, then $v_{k} \stackrel{l-k}{\longrightarrow} v_{l} \rightarrow v_{k}$ is a directed cycle of length $l-k+1$, and hence $l=k+n-1$ or $l=k+n$. So $(k, l)$ is $(0, n-1),(0, n)$ or $(1, n)$. When $(k, l)$ is $(0, n-1),\left(y_{1}, y_{2}\right)$ is $\left(v_{0}, w_{0}\right)$ and when $(k, l)$ is $(1, n)$, $\left(y_{1}, y_{2}\right)$ is $\left(v_{0}, w_{0}\right)$ or $\left(w_{0}, v_{0}\right)$. If $\left(v_{k}, v_{l}\right) \in A_{E}$, then $v_{k} \rightarrow v_{l} \stackrel{n+1-l}{\longrightarrow} v_{0} \stackrel{k}{\longrightarrow} v_{k}$ is a directed cycle of length $n-l+k+2$, and hence $l=k+1$ or $l=k+2$. Let $l=k+2$. If $k \geq 1$, then $\left(y_{1} . y_{2}\right)$ does not exist. So $(k, l)$ is $(0,2)$ and $\left(y_{1}, y_{2}\right)$ is $\left(w_{0}, v_{0}\right)$. In conclusion, there are two possible forms of digraphs. Firstly, when $\left(y_{1}, y_{2}\right)$ $=\left(v_{0}, w_{0}\right)$, the only arcs which might be added to $\left(A_{0}\right)_{n+2} \cup\left\{\left(w_{0}, v_{1}\right)\right\}$ are $\left(w_{0}, v_{2}\right),\left(v_{n-1}, v_{0}\right)$, and $\left(v_{n}, v_{1}\right)$. As $B_{n+2}^{\sim}=\left\{\left(w_{0}, v_{1}\right),\left(w_{0}, v_{2}\right),\left(v_{n-1}, v_{0}\right),\left(v_{n}\right.\right.$, 
$\left.\left.v_{1}\right)\right\}, E$ is a subgraph of $\tilde{E_{n+2}}$. Secondly, when $\left(y_{1}, y_{2}\right)=\left(w_{0}, v_{0}\right)$, the only arcs which might be added to $\left(A_{0}\right)_{n+2} \cup\left\{\left(w_{0}, v_{1}\right)\right\}$ are $\left(v_{n-1}, w_{0}\right),\left(v_{n}, v_{1}\right)$, and $\left(v_{0}, v_{2}\right)$. If we switch $v_{0}$ and $w_{0}$, we get the same arcs as above and hence $E$ is a subgraph of ${E_{n+2}}^{\sim}$.

Case (ii)b: $j=2$.

If $\left(w_{0}, v_{1}\right) \in A_{E}$, then it reduces to Case (ii)a. Assume $\left(w_{0}, v_{1}\right) \notin A_{E}$. As there can be only directed cycles of order $n$ or $n+1$ in $E$, among the arcs which are to $w_{0}$, only $\left(v_{0}, w_{0}\right)$ may exist. Assume $\left(v_{0}, w_{0}\right) \in A_{E}$. After exchanging $v_{i}$ with $v_{n+2-i}$ for $2 \leq i \leq n$, and $v_{0}$ with $v_{1}$, we get $E^{T}$ where $E$ is described in Case (ii)a which has arc $\left(w_{0}, v_{2}\right)$ and arcs of $\left(A_{0}\right)_{n+2} \bigcup\left\{\left(w_{0}, v_{1}\right)\right\}$. Thus by similar argument, $E^{T}$ is a subgraph of $E_{n+2}^{\tilde{2}}$. Assume $\left(v_{0}, w_{0}\right) \notin A_{E}$. As there can be only directed cycles of order $n$ or $n+1$ in $E$, among the arcs which are from $w_{0}$, only $\left(v_{n}, w_{0}\right)$ may exist where $\left(y_{1}, y_{2}\right)$ is $\left(v_{0}, w_{0}\right)$ or $\left(w_{0}, v_{1}\right)$. Let $0 \leq k<l \leq n$. If $\left(v_{l}, v_{k}\right) \in A_{E}$ and $(k, l) \neq(0, n)$, then by the same argument as in Case (ii)a, $(k, l)$ is $(0, n-1)$, or $(1, n)$ and $\left(y_{1}, y_{2}\right)$ is $\left(v_{0}, w_{0}\right)$. If $\left(v_{k}, v_{l}\right) \in A_{E}$ where $l \neq k+1$, then by the same argument as in Case (ii)a, $(k, l)$ is $(0,2)$ or $(1,3)$ and $\left(y_{1}, y_{2}\right)$ is $\left(w_{0}, v_{1}\right)$. In conclusion, there are two possible forms of digraphs. Firstly when $\left(y_{1}, y_{2}\right)=\left(v_{0}, w_{0}\right)$, the only arcs which might be added to $\left(A_{0}\right)_{n+2} \cup\left\{\left(w_{0}, v_{2}\right)\right\}$ are $\left(v_{n-1}, v_{0}\right)$ and $\left(v_{n}, v_{1}\right)$ and hence $E$ is a subgraph of $E_{n+2}$. Secondly when $\left(y_{1}, y_{2}\right)=\left(w_{0}, v_{1}\right)$, the only arcs which might be added to $\left(A_{0}\right)_{n+2} \cup\left\{\left(w_{0}, v_{2}\right)\right\}$ are $\left(v_{0}, v_{2}\right)$ and $\left(v_{1}, v_{3}\right)$. After exchanging $v_{i}$ with $v_{n+2-i}$ for $2 \leq i \leq n$ and $v_{0}$ with $v_{1}, E^{T}$ is a subgraph of $E_{n+2}^{\sim}$.

\section{References}

[1] A. Brauer, On a problem of partitions, Amer. J. Math. 64 (1942), 299-312.

[2] B. M. Kim, B. C. Song, and W. Hwang, Wielandt type theorem for Cartesian product of digraphs, Linear Algebra Appl. 429 (2008), no. 4, 841-848.

[3] R. Lamprey and B. Barnes, Primitivity of products of digraphs, Proceedings of the Tenth Southeastern Conference on Combinatorics, Graph Theory and Computing (Florida Atlantic Univ., Boca Raton, Fla., 1979), pp. 637-644, Congress. Numer., XXIII-XXIV, Utilitas Math., Winnipeg, Man., 1979.

[4] B. L. Liu, B. D. McKay, N. C. Wormald, and K. Zhang, The exponent set of symmetric primitive $(0,1)$ matrices with zero trace, Linear Algebra Appl. 133 (1990), 121-131.

[5] J. B. Roberts, Note on linear forms, Proc. Amer. Math. Soc. 7 (1956), 465-469.

[6] J. Y. Shao, The exponent set of symmetric primitive matrices, Sci. Sinica Ser. A 9 (1986), 931-939.

[7] H. Wielandt, Unzerlegbare, nicht negative Matrizen, Math. Z. 52 (1950), 642-648.

ByEONG MOON KIM

Department of Mathematics

Gangneung-Wonju National University

Gangneung 210-702, Korea

E-mail address: kbm@kangnung.ac.kr 
Yoomi Rho

Department of Mathematics

UNIVERSITY OF INCHEON

INCHEON 406-772, KOREA

E-mail address: rho@incheon.ac.kr 